\title{
The effect of simulated acid rain on microbial community structure in decomposing leaf litter
}

\author{
Sangsub Cha ${ }^{1}$, Sung-Min Lim ${ }^{1}$, Bahitkul Amirasheba ${ }^{2}$, Jae-Kuk Shim ${ }^{1, *}$ \\ ${ }^{1}$ Department of Life Science, Chung-Ang University, Seoul 156-756, Korea \\ ${ }^{2}$ Kazakh National Pedagogical University Named After Abay, 050010 Almaty, Kazakhstan
}

\begin{abstract}
Acid deposition is one of the most serious environmental problems in ecosystems. The present study surveyed the effects of simulated acid rain on leaf litter mass loss and microbial community in the decomposing leaf litter of Sorbus anifolia in a microcosm at $23^{\circ} \mathrm{C}$ and $40 \%$ humidity. Microbial biomass was measured by substrate-induced respiration (SIR) and phospholipid fatty acids (PLFAs), and the microbial community structures were determined by composition of PLFAs at each interval of decomposition in litter sample and at each $\mathrm{pH}$ treatment. The microbial biomass showed peaks at midstage of decomposition, decreasing at the late stage. The leaf litter mass loss of S. anifolia decreased with decreasing $\mathrm{pH}$ during early and mid- decomposition stages; however the mass loss becomes similar between pH treatments at late-decomposition stage. The acidification remarkably lowers the microbial biomass of bacteria and fungi; however, microbial diversity was unchanged between $\mathrm{pH}$ treatments at each stage of litter decomposition. With changes of decomposition stage and pH treatment there were considerable differences in replacement and compensation of microbial species. Fungi/bacteria ratio was considerably changed by $\mathrm{pH}$ treatment. The PLFA profile showed significantly larger fungi/bacteria ratio at $\mathrm{pH} 5$ than $\mathrm{pH} 3$ at the early stage of decomposition, and the difference becomes smaller at the later decomposition stage. At low pH, pH 3 and pH 4, the fungi/bacteria ratios were stable according to the litter decomposition stages.

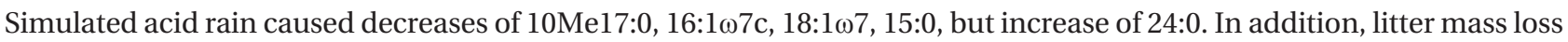
showed significant positive correlation with microbial biomass measured by SIR and PLFA on the decomposing leaf litter.
\end{abstract}

Key words: acid rain, litter decomposition, microbial biomass, PLFA

\section{INTRODUCTION}

Acidification of precipitation caused by human activities has become one of the most serious problems in natural ecosystems (Berg and Laskowski 2006). Acid rain influences on terrestrial plants performances include cation-loss or nutrient-loss from leaves (Proctor 1983, Liu et al. 2007), change in the gas-exchange rate through stomata on leaves (Evans et al. 1982, Hermle et al. 2007), germination (Lee and Weber 1979, Raynal et al. 1982), and plant growth (Wood and Bormann 1974, Neufold et al. 1985, Reich et al. 1987). In addition, acid rain is known to change the soil chemical status and function of the decomposer community, often leading to imbalances in nutrient cycling, litter decomposition, and productivity of the ecosystem (Pennanen et al. 1998). It resulted in poor soil fertility, and the harmful effects on plant growth by leaching of toxic soluble Al (Wood et al. 1984, Matzner et al. 1986, Osonubi et al. 1988), and releases such as Ca, Mg, and $\mathrm{K}$ from the solid phases of soil into soil solution (Batty http://dx.doi.org/10.5141/ecoenv.2013.223

(c) 1 This is an Open Access article distributed under the terms of
the Creative Commons Attribution Non-Commercial Licens
(http:// creativecommons.org/licenses/by-nc/3.0/) which
permits unrestricted non-commercial use, distribution, and reproduction in any
medium, provided the original work is properly cited.
Received 31 August 2013, Accepted 18 November 2013

*Corresponding Author

E-mail: shimjk@cau.ac.kr Tel: +82-2-820-5211 
and Younger 2007, Zhang et al. 2007).

In terrestrial ecosystems, the litter decomposition carried out by microorganisms is one of the most important processes in decomposer subsystem. In particular, strong reductions in microbial activity are easily detected when low pH acid rain is applied (Bååth et al. 1979, Bewley and Parkinson 1985, Fritze 1992, Myrold and Nalson 1992, Rousk et al. 2009). The essential nutrient ( $\mathrm{K}$ and $\mathrm{Mg}$ ) released from plant litter were found to be affected by low pH (Holvland et al. 1980, Batty and Younger 2007, Berg and Laskowski 2006), while decomposition of plant litter showed no significant effect of the acid rain. In addition, Ouyang et al. (2008) suggested the mineralization of soil organic $\mathrm{C}$ was not related to $\mathrm{pH}$ level. On the other hand, Berg and Laskowski (2006) demonstrated that such changes in litter, leaving larger recalcitrant remains, may lead to changed decomposition patterns, which should decrease the extent of organic matter decomposition in ecosystems and cause a higher humus accumulation rate.

Most studies on the effect of acid rain noted that the species of acid-tolerant fungi and bacteria have shown increased activity in plant litter decomposition in terrestrial environments (Gross and Robbins 2000, Reith et al. 2002), and the effects of the $\mathrm{pH}$ gradient on microbial community structure and the patterns were demonstrated (Frostegård et al. 1993, Bååth and Arnebrant 1994, Bååth et al. 1992, 1995, Pennanen et al. 1998, Bååth and Anderson 2003, Stemmer et al. 2007, Högberg et al. 2007, Rousk et al. 2009).

Several techniques have been used to identify fungal and bacterial groups in soil. One of the most commonly used methods is the selective inhibition technique (Anderson and Domsch 1973) based on inhibition of the substrate-induced respiration (SIR) using antibiotics that inhibit bacterial and fungal respiration (Bååth and Anderson 2003), thymidine incorporation technique (Bååth and Arnebrant 1994), and by analysis the ester-linked phospholipid fatty acids (PLFAs) composition of the soil, since different subsets of the community have different PLFA patterns (Bååth et al. 1995). Frostegård and Bååth (1996) suggested that a fungal/bacterial biomass ratio could be estimated by using the PLFA 18:2v6,9 as a measure of fungal biomass and the sum of 13 bacteria specific PLFAs as a measure of bacterial biomass (Bååth and Anderson 2003, Frostegård et al. 1993).

The aim of the present study was to assess the effect of simulated acid rain on plant litter decomposition processes in a microcosm environment. The leaf litter samples were treated periodically with simulated acid rain, which was made using sulfuric acid, and retrieved for the determination of weight loss and microbial biomass, microbial community structure, and microbial activities on the decomposing litter. Microbial biomass and microbial community structures on the leaf litter were determined by SIR and PLFA methods.

\section{MATERIALS AND METHODS}

\section{Microcosm Design and Litter Decomposition}

Sorbus alnifolia is known to have a wide tolerance range to atmospheric pollution and has a trend of increasing distributional area near big cities in Korea (You et al. 1998). We collected freshly fallen leaf litter of S. alnifolia at autumn, dried at $60^{\circ} \mathrm{C}$ dry oven, and cut to $2 \mathrm{~cm}$ width for easy use in the microcosm.

The study used $1 \mathrm{~L}$ colorless glass bottles with detachable lids which have $9 \mathrm{~mm}$ diameter holes filled with $300 \mathrm{~g}$ of clean quartz sand. The quartz sand contained $90 \%$ water holding capacity by adding deionized water to maintain constant moisture in the microcosm bottles. Approximately $4 \mathrm{~g}$ of litter samples were soaked in distilled water for $1 \mathrm{~h}$, and then placed on top of the sand in the microcosm glass bottle, and incubated at constant $23^{\circ} \mathrm{C}$ and $40 \%$ relative humidity.

The simulated acid rain of $\mathrm{pH} 3, \mathrm{pH} 4$, and $\mathrm{pH} 5$ were made by dilution of sulfuric acid in deionized water. After 16 days of $10 \mathrm{ml}$ fresh soil suspension solution addition, $10 \mathrm{ml}$ of simulated acid rain of each $\mathrm{pH}$ level applied to each experimental treatment group, and additional artificial acid rain water supplied at twice a week as much as the same amount of evaporated water from the microcosm until the end of experiment. The four replicated litter samples were carefully retrieved from microcosm after 80 and 160, and 290 experimental days. Retrieval fresh litter samples from microcosm use to measure the mass loss measurement and microbial biomass and microbial community analysis.

\section{Microbial Biomass}

Fungal, bacterial and total microbial biomass $\mathrm{C}\left(\mathrm{C}_{\text {mic }}\right)$ were determined by the SIR technique of Anderson and Domsch (1978) with modifications by Beare et al. (1990). Four subsamples (0.5-1.0 g dry weight equivalent) of each litter sample were weighed into $100 \mathrm{ml}$ serum bottles and incubated at $4^{\circ} \mathrm{C}$ for $12 \mathrm{~h}$ with $2.5 \mathrm{ml}$ microbial respiration inhibitor per gram of litter sample. Cycloheximide (16.0 $\mathrm{g} / \mathrm{l})$ and streptomycin $(3.2 \mathrm{~g} / \mathrm{l})$ for fungal and bacterial 
respiratory inhibitors were used, respectively. Deionized water was added instead of microbial inhibitors for control treatment. All treatments had $2.5 \mathrm{ml} / \mathrm{g}$ glucose solutions $(16.0 \mathrm{~g} / \mathrm{l})$ added as a substrate and were immediately flushed with $\mathrm{CO}_{2}$-free gas, sealed tightly, and incubated at $23^{\circ} \mathrm{C}$ for $2 \mathrm{~h}$. The $\mathrm{CO}_{2}$ concentrations in the bottles were measured by infrared gas analyzer (IRGA) after incubation.

Microbial biomass- $\mathrm{C}$ was calculated according to the equation of Beare et al. (1990) as follows:

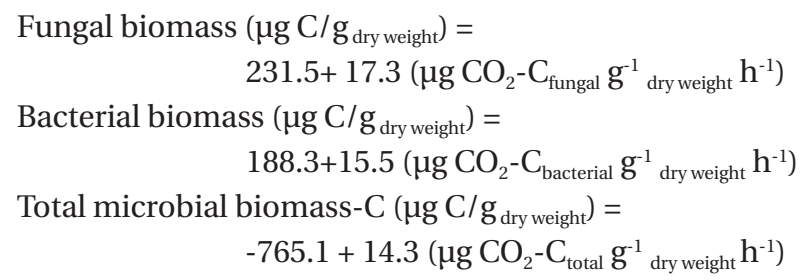

\section{Microbial Community Structure}

PLFAs were extracted and analyzed according to the procedure described by Wilkinson et al. (2002). Fresh litter samples were freeze-dried and ground to less than $10 \mu \mathrm{m}$ by blender. $250 \pm 1 \mathrm{mg}$ of freeze-dried litter sample was extracted by addition of $1.6 \mathrm{ml}$ of potassium phosphate $(50 \mathrm{mmol} / \mathrm{l}, \mathrm{pH} 7.4)$, methanol $(4 \mathrm{ml})$ and chloroform $(2 \mathrm{ml})$ in $25 \mathrm{ml}$ glass tubes. The tubes were vortexed for $30 \mathrm{~s}$ and sonicated for $2 \mathrm{~min}$ prior to incubation in a water bath at $37^{\circ} \mathrm{C}$ for $30 \mathrm{~min}$. The tubes were shaken for $2 \mathrm{~h}$ horizontally, and then the supernatant liquid transferred to a $50 \mathrm{ml}$ separatory funnel, and chloroform (4 ml) and water $(4 \mathrm{ml})$ were added to the separating funnel. The chloroform phase was collected, reduced in volume by rotary evaporation, and fractionated by chromatography on silicic acid (mesh size 200-400). Neutral lipids and glycolipids were eluted with chloroform $(3 \mathrm{ml} \times 2)$ and acetone ( $3 \mathrm{ml} \times 2)$, respectively. Polar lipids were eluted with $3 \mathrm{ml} \times 2$ methanol and collected in glass test tubes. The solvent was evaporated under a stream of oxygen-free $\mathrm{N}_{2}$, and the residue subjected to alkaline methanolysis by vortexing for $30 \mathrm{~s}$ in a mixture of $2 \mathrm{ml}$ of $0.2 \mathrm{M}$ methanolic $\mathrm{KOH}$ and toluene:methanol $(1: 1, \mathrm{v} / \mathrm{v})$ solution, and followed by incubation at $37^{\circ} \mathrm{C}$ for $30 \mathrm{~min}$. After cooling, the mixtures were neutralized with $1 \mathrm{~mol}$ acetic acid and fatty acid methyl esters (FAMEs) extracted twice by $2 \mathrm{ml}$ of chloroform:hexane $(1: 4, v / v)$. After evaporation of the solvent under oxygen-free $\mathrm{N}_{2}$, the FAMEs were re-suspended in chloroform:hexane (1:4, v/v) containing $15 \mu \mathrm{g}$ nonadecanoic acid methyl ester (Sigma Chemical, Poole, UK) as an internal standard. FAMEs were quantified by gas chromatography mass spectrometry; Hewlett Packard HP6890 Gas Chromatograph (Hewlett Packard, Palo Alto, CA, USA) equipped with a DB-5MS column $(0.2 \mathrm{~mm}$ [inner diameter] by $25 \mathrm{~m} ; 0.25 \mu \mathrm{m}$ film thickness). A splitless injection was employed (injector at $300^{\circ} \mathrm{C}$ ), and the oven was maintained at $60^{\circ} \mathrm{C}$ for $1 \mathrm{~min}$ after injection. The oven temperature was then increased to $150^{\circ} \mathrm{C}$ at $30^{\circ} \mathrm{C} /$ min and maintained for $4 \mathrm{~min}$, followed by an increase to $250^{\circ} \mathrm{C}$ at $4^{\circ} \mathrm{C} / \mathrm{min}$ and held for $15 \mathrm{~min}$. Finally, the oven temperature was increased to $300^{\circ} \mathrm{C}$ at $25^{\circ} \mathrm{C} / \mathrm{min}$ and held for $6 \mathrm{~min}$. The transfer line was held at $280^{\circ} \mathrm{C}$ throughout. Helium was used as the carrier gas $(0.8 \mathrm{ml} / \mathrm{min})$. FAMEs were identified by retention time and/or electron ionization mass spectrometry, HP6890 Mass Selective Detector (Hewlet Packard).

Bacterial biomass was estimated from the summed concentrations of the following PLFAs: $i 15: 0, a 15: 0,15: 0$, $i 16: 0,16: 1 \omega 9,16: 1 \omega 7 \mathrm{t}, i 17: 0, a 17: 0,17: 0$, cy17:0, 18:1 $\omega 7$ and cy19:0 (Frostegård and Bååth 1996, Pennanen et al. 1998, 1999, Bååth and Anderson 2003). Fungal biomass was estimated from concentrations of the marker 18:2 $\omega 6,9$ (Federle 1986, Frostegård and Bååth 1996, Pennanen et al. 1998, 1999, Bååth and Anderson 2003).

\section{Microbial Diversity}

The Shannon-Wiener index (Shannon 1948), Simpson's diversity (Simpson 1949) and Evenness were generally defined as follows: where $H$ was Shannon-Wiener index, $J$ was Evenness, $D_{s}$ was Simpson Diversity, s was the total number of FAMEs in each sample and $p_{i}$ was the percentage of the peak area of $\mathrm{FAME}_{\mathrm{i}}$ to the total area of each sample.

$$
\begin{aligned}
& H=-\sum_{i=1}^{s} P_{i} \log P_{i} \\
& J=H / H_{\max } \\
& H_{\max }=\log (s) \\
& D_{s}=1-\sum_{i=1}^{s}\left(P_{i}\right)^{2}
\end{aligned}
$$

\section{Statistical Analysis}

Differences between samples, in regard to litter mass loss, and microbial biomass were analyzed statistically using a one-way ANOVA followed by a Turkey's Honestly Significant Difference (HSD) test. For each value, linear correlations of coefficients were provided. All statistical work was performed with SPSS ver. 17.0 (SPSS Inc., Chicago, IL, USA). 


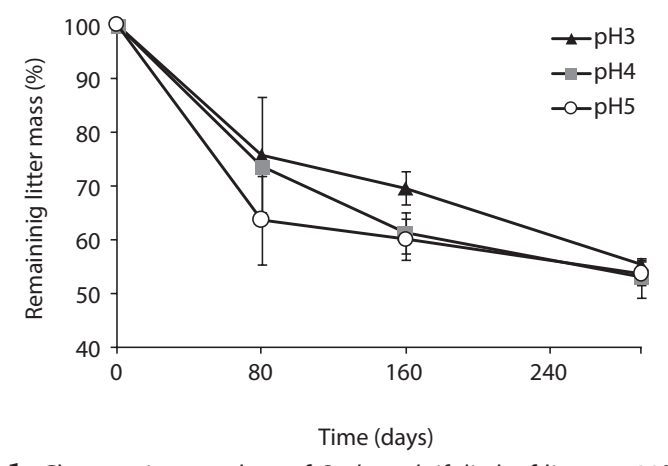

Fig. 1. Changes in mass loss of Sorbus alnifolia leaf litter at $23^{\circ} \mathrm{C}$ and constant humidity microcosm in the different $\mathrm{pH}$ of simulated acid rain treatments. Vertical lines indicate standard deviation $(n=4)$.

\section{RESULTS}

\section{Litter Mass Loss}

The litter decomposition was the slowest at $\mathrm{pH} 3$ treatment, and the most rapid at pH 5 treatment in the early and mid-stage of decomposition (Fig. 1). In the early stage of litter decomposition, mass loss at $\mathrm{pH} 5$ was $38.5 \%$, and $26.7 \%$ at $\mathrm{pH}$. The decomposition rate between $\mathrm{pH} 3$ and pH 5 showed significant difference $(P=0.001)$; however, there is no significant difference in the late stage between each $\mathrm{pH}$ treatment.

\section{Microbial Biomass Determined by SIR}

The microbial biomass, determined by SIR method, of decomposing litter samples treated with different acidity of artificial rain are presented in Fig. 2. The microbial biomass decreased with increasing acidity, showing the smallest at pH 3 and the largest at pH 5 treatment. The microbial biomass increased with decomposition proceeds and showed peaks at mid-stage decomposition and decreased at the late stage.

After 160 days decomposition, the mid-stage of decomposition, total microbial biomass- $\mathrm{C}$ was measured as $12.97 \mathrm{mg} \mathrm{C}_{\text {mic }} / \mathrm{g}$ litter at $\mathrm{pH} \mathrm{3,17.39} \mathrm{mg} \mathrm{C}_{\text {mic }} / \mathrm{g}$ litter at $\mathrm{pH} \mathrm{4}$, and $23.25 \mathrm{mg} \mathrm{C}_{\text {mic }} / \mathrm{g}$ litter at $\mathrm{pH}$ 5. Bacterial biomass increased from $4.95 \mathrm{mg} \mathrm{C}_{\text {mic }} / \mathrm{g}$ litter to $8.94 \mathrm{mg} \mathrm{C}_{\text {mic }} / \mathrm{g}$ litter from $\mathrm{pH} 3$ to $\mathrm{pH} 5$ at 80 days, and $4.88 \mathrm{mg} \mathrm{C}_{\text {mic }} / \mathrm{g}$ litter to $11.57 \mathrm{mg} \mathrm{C}_{\text {mic }} / \mathrm{g}$ litter at $\mathrm{pH} 3$ to $\mathrm{pH} 5$ at 160 days after treatment. Fungal biomass increased from 5.69 to $6.92 \mathrm{mg}$ $\mathrm{C}_{\text {mic }} / \mathrm{g}$ litter from $\mathrm{pH} 3$ to $\mathrm{pH} 5$ at 80 days, and then, after 80 days from 8.38 to $11.85 \mathrm{mg} \mathrm{C}_{\text {mic }} / \mathrm{g}$ litter from $\mathrm{pH} 3$ to pH 5. Unlike fungal biomass, bacterial biomass sharply increased at the initial decomposition stage, and slowly decreased or plateaued after the early stage of decomposition in low $\mathrm{pH}$ treatments.

Consequently, in the early decomposition period, the fungal biomass was the same as the bacterial biomass, especially at low $\mathrm{pH}$; however, the fungal biomass dominated on the decomposing litter at the mid-decompositional stage. Although bacterial and fungal biomass decreased with decreasing acidity, the simulated acid rain affected bacterial biomass more than it did fungal biomass. In other words, fungal biomass was more stable in acidic conditions than bacterial biomass.

\section{Microbial Community Structure on the Decaying Leaf Litter}

Microbial PLFA profiles of decomposing S. alnifolia leaf litter that were treated with different $\mathrm{pH}$ of artificial acid rain are shown in Table 1. Changes of the fungal, bacterial, and total microbial PLFAs on the decomposing litter samples are presented in Fig. 3. The changes in PLFAs of fungi showed similar patterns to the change of microbial biomass determined by SIR technique. The bacterial and fungal PLFAs concentration decreased remarkably with increasing acidity of artificial rain, and showed peak concentration at the mid-stage of litter decomposition at all microbial groups. The concentration of bacterial PLFAs was 1.4 to 2.9 times greater than that of fungal PLFAs.

Each PLFA marker showed a different pattern of responses at different $\mathrm{pH}$ treatments and decomposition stages. PLFAs of gram positives almost linearly increased with increasing $\mathrm{pH}$; however, the PLFAs of gram negatives also showed increasing patterns at the early and midstages of decomposition then rapidly decreased at the late stage (Fig. 4).

As the process of $S$. alnifolia litter decomposition, the

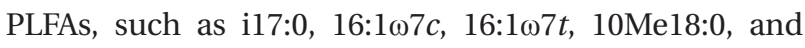
20:4, markedly increased at mid and late-stages in the early stage, and the i15:0, i16:0, a17:0, and 15:0 showed increases. However, the PLFA of 16:1 $\omega 5$ and 24:0 decreased as decomposition proceeded. Most PLFA markers showed peaks at mid-stage of decomposition, and then decreased at the late-stage decomposition. In late stage of litter de-

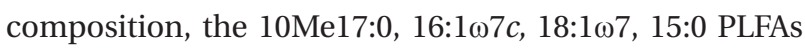
increased with increasing $\mathrm{pH}$ value, while 24:0 showed the opposite pattern.

\section{Microbial Diversity}

The number of PLFAs on the decomposing leaf litter samples was increased with decomposition processed 

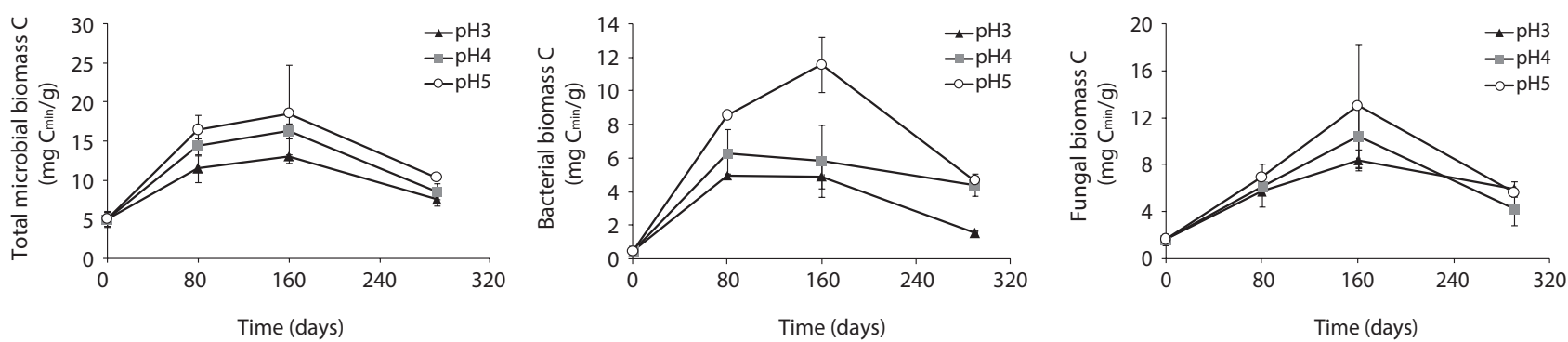

Fig. 2. Total microbial biomass $\mathrm{C}\left(\mathrm{mg} \mathrm{C}_{\text {mid }} / \mathrm{g}\right)$, bacterial biomass, and fungal biomass measured by substrate induced respiration (SIR) method at different $\mathrm{pH}$ levels of artificial acid rain treated decomposing leaf litter of Sorbus alnifolia. The error bars indicate standard deviation $(n=4)$.
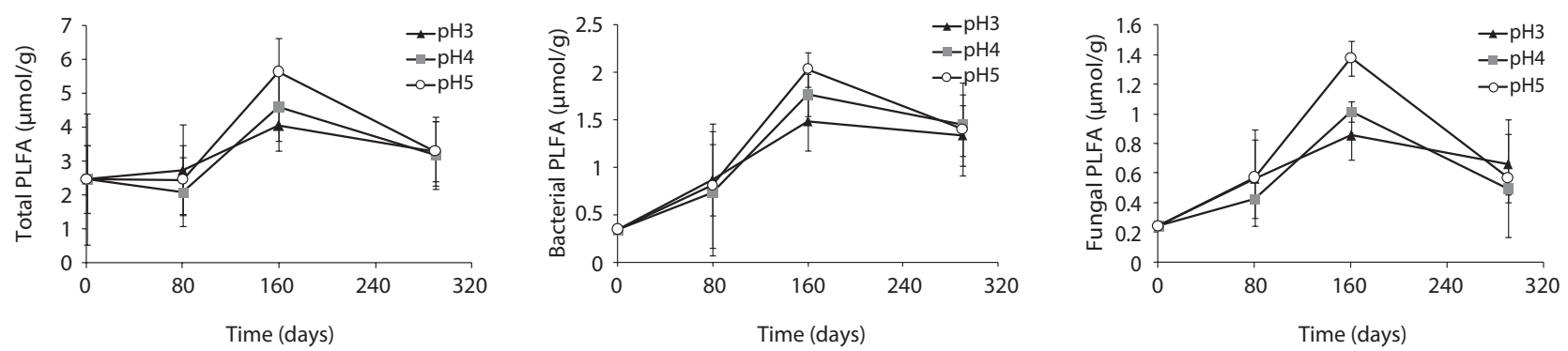

Fig. 3. Total, bacterial, fungal PLFAs in each artificial acid rain treatment of Sorbus alnifolia litter samples during decomposition in microcosm condition. The error bars indicate standard deviation $(n=4)$.
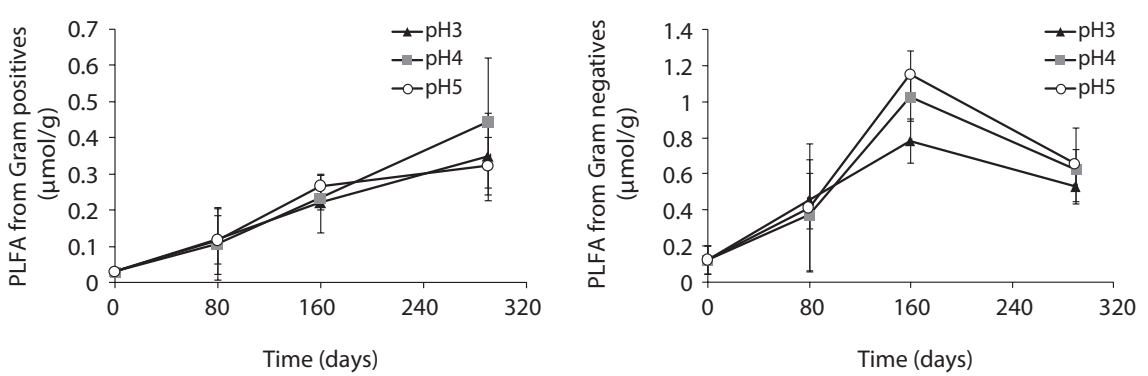

Fig. 4. Changes in PLFAs of Gram positives and Gram negatives according to the Sorbus alnifolia litter decomposition in the microcosm. The error bars indicate standard deviation $(n=4)$.

(Table 2). Fourteen kinds of PLFAs were presenting the initial decomposition litter sample just before the artificial rain treatment. The number of PLFAs was increased to 22 after 80 days, 27 after 160 days, and 26-27 after 290 days. However, the diversity indices and evenness indices at different $\mathrm{pH}$ treatment and between litter decomposition stages showed no significant differences although Simpson diversity indices slightly increased with decomposition time elapsed. These results suggested that there were large amounts of displacement of microbial taxa according to $\mathrm{pH}$ treatment and proceeds of litter decomposition.

\section{DISCUSSION}

Litter decomposition mainly performed by soil microbial activities plays very important roles such as nutrient cycling in the natural ecosystems. In addition, many processes of litter decomposition were influenced by physicochemical environments and chemical contents of leaf litter (Heal et al. 1997, Zimmer 2002, Sariyildiz and Anderson 2003).

The result of this study showed a remarkable decrease of leaf litter mass loss especially at early and mid-stages of decomposition with artificial acid rain treatment, con- 
Table 1. PLFA profiles of the decomposing S. alnifolia litter samples treated with simulated acid rain in the microcosm. Values are means of total PLFA concentrations (nmol/g), standard deviation in parentheses, and n.m is not measurable

\begin{tabular}{|c|c|c|c|c|c|c|c|c|c|c|c|}
\hline & & \multirow{3}{*}{ Initial } & \multicolumn{9}{|c|}{ PLFA concentration ( $\mathrm{nmol} / \mathrm{g})$} \\
\hline & & & \multicolumn{3}{|c|}{ After 80 days } & \multicolumn{3}{|c|}{ After 160 days } & \multicolumn{3}{|c|}{ After 290 days } \\
\hline & & & pH3 & $\mathrm{pH} 4$ & pH5 & pH3 & $\mathrm{pH} 4$ & pH5 & pH3 & $\mathrm{pH} 4$ & pH5 \\
\hline \multicolumn{12}{|l|}{ Gram + } \\
\hline & i15:0 & $\begin{array}{l}31.1 \\
(4.9)\end{array}$ & $\begin{array}{c}22.4 \\
(11.1)\end{array}$ & $\begin{array}{c}13.9 \\
(11.7)\end{array}$ & $\begin{array}{c}37.9 \\
(4.88)\end{array}$ & $\begin{array}{c}94.3 \\
(35.5)\end{array}$ & $\begin{array}{c}107.9 \\
(14)\end{array}$ & $\begin{array}{c}96.5 \\
(18.7)\end{array}$ & $\begin{array}{l}131.3 \\
(6.29)\end{array}$ & $\begin{array}{l}154.4 \\
(7.71)\end{array}$ & $\begin{array}{l}84.2 \\
(6.2)\end{array}$ \\
\hline & a15:0 & n.m & $\begin{array}{c}26.0 \\
(11.8)\end{array}$ & $\begin{array}{c}26.8 \\
(14.7)\end{array}$ & $\begin{array}{l}23.1 \\
(9.6)\end{array}$ & $\begin{array}{c}34.1 \\
(20.7)\end{array}$ & $\begin{array}{c}40.5 \\
(11.5)\end{array}$ & $\begin{array}{c}58.0 \\
(12.4)\end{array}$ & $\begin{array}{c}41.7 \\
(11.1)\end{array}$ & $\begin{array}{c}61.8 \\
(27.9)\end{array}$ & $\begin{array}{c}41.5 \\
(28.9)\end{array}$ \\
\hline & i16:0 & n.m & $\begin{array}{l}15.1 \\
(9.5)\end{array}$ & $\begin{array}{c}9.4 \\
(10.0)\end{array}$ & $\begin{array}{c}16.2 \\
(19.1)\end{array}$ & $\begin{array}{c}38.1 \\
(14.2)\end{array}$ & $\begin{array}{l}30.2 \\
(5.6)\end{array}$ & $\begin{array}{l}39.7 \\
(6.5)\end{array}$ & $\begin{array}{c}49.2 \\
(20.1)\end{array}$ & $\begin{array}{l}65.8 \\
(25)\end{array}$ & $\begin{array}{l}53.6 \\
(12)\end{array}$ \\
\hline & 10Me16:0 & n.m & $\begin{array}{c}30.7 \\
(19.4)\end{array}$ & $\begin{array}{c}28.3 \\
(32.5)\end{array}$ & $\begin{array}{l}13.0 \\
(9.0)\end{array}$ & $\begin{array}{l}16.8 \\
(8.5)\end{array}$ & $\begin{array}{l}19.5 \\
(2.9)\end{array}$ & $\begin{array}{l}17.9 \\
(3.6)\end{array}$ & $\begin{array}{l}53.4 \\
(8.1)\end{array}$ & $\begin{array}{l}71.1 \\
(27)\end{array}$ & $\begin{array}{c}60.5 \\
(13.5)\end{array}$ \\
\hline & i17:0 & n.m & n.m & n.m & n.m & $\begin{array}{l}15.0 \\
(5.9)\end{array}$ & $\begin{array}{l}14.9 \\
(1.7)\end{array}$ & $\begin{array}{l}14.5 \\
(1.4)\end{array}$ & $\begin{array}{l}22.0 \\
(9.6)\end{array}$ & $\begin{array}{l}25.7 \\
(7.2)\end{array}$ & $\begin{array}{l}25.1 \\
(5.6)\end{array}$ \\
\hline & a17:0 & n.m & $\begin{array}{l}15.0 \\
(8.9)\end{array}$ & $\begin{array}{c}18.0 \\
(13.4)\end{array}$ & $\begin{array}{l}14.9 \\
(3.9)\end{array}$ & $\begin{array}{l}12.5 \\
(3.8)\end{array}$ & $\begin{array}{l}12.7 \\
(4.3)\end{array}$ & $\begin{array}{l}16.8 \\
(1.9)\end{array}$ & $\begin{array}{c}42.1 \\
(12.1)\end{array}$ & $\begin{array}{c}51.7 \\
(11.3)\end{array}$ & $\begin{array}{l}42.3 \\
(9.4)\end{array}$ \\
\hline & 10Me17:0 & n.m & $\begin{array}{l}12.5 \\
(8.6)\end{array}$ & $\begin{array}{c}12.0 \\
(16.8)\end{array}$ & $\begin{array}{l}7.4 \\
(3.6)\end{array}$ & $\begin{array}{c}9.8 \\
(1.3)\end{array}$ & $\begin{array}{l}10.3 \\
(0.8)\end{array}$ & $\begin{array}{c}23.4 \\
(11.3)\end{array}$ & $\begin{array}{c}8.7 \\
(1.8)\end{array}$ & $\begin{array}{l}12.6 \\
(4.6)\end{array}$ & $\begin{array}{l}15.8 \\
(3.5)\end{array}$ \\
\hline & Subtotal & 31.1 & 121.7 & 108.4 & 112.5 & 220.6 & 235.9 & 266.8 & 348.5 & 443.2 & 322.9 \\
\hline \multicolumn{12}{|l|}{ Gram - } \\
\hline & $16: 1 \omega 7 \mathrm{c}$ & n.m & n.m & n.m & n.m & $\begin{array}{l}173.9 \\
(46.2)\end{array}$ & $\begin{array}{l}218.6 \\
(26.9)\end{array}$ & $\begin{array}{l}269.6 \\
(34.9)\end{array}$ & $\begin{array}{l}124.0 \\
(24.2)\end{array}$ & $\begin{array}{l}149.7 \\
(38.3)\end{array}$ & $\begin{array}{l}169.3 \\
(43.4)\end{array}$ \\
\hline & cy17:0 & $\begin{array}{c}20.3 \\
(23.6)\end{array}$ & $\begin{array}{l}36.1 \\
(6.8)\end{array}$ & $\begin{array}{c}19.6 \\
(23.4)\end{array}$ & $\begin{array}{l}18.5 \\
(9.1)\end{array}$ & $\begin{array}{l}25.5 \\
(6.3)\end{array}$ & $\begin{array}{l}31.9 \\
(2.0)\end{array}$ & $\begin{array}{c}43.4 \\
(12.0)\end{array}$ & $\begin{array}{l}25.1 \\
(7.2)\end{array}$ & $\begin{array}{l}30.8 \\
(7.3)\end{array}$ & $\begin{array}{c}26.2 \\
(11.4)\end{array}$ \\
\hline & $18: 1 \omega 7$ & $\begin{array}{c}91.0 \\
(42.8)\end{array}$ & $\begin{array}{c}383.1 \\
(126.7)\end{array}$ & $\begin{array}{c}329.1 \\
(266.6)\end{array}$ & $\begin{array}{c}295.5 \\
(216.7)\end{array}$ & $\begin{array}{l}489.5 \\
(57.7)\end{array}$ & $\begin{array}{c}687.4 \\
(103.6)\end{array}$ & $\begin{array}{l}745.8 \\
(90.0)\end{array}$ & $\begin{array}{l}312.9 \\
(42.1)\end{array}$ & $\begin{array}{l}371.2 \\
(68.0)\end{array}$ & $\begin{array}{c}411.2 \\
(129.5)\end{array}$ \\
\hline & cy19:0 & $\begin{array}{c}12.9 \\
(12.6)\end{array}$ & $\begin{array}{c}33.9 \\
(19.7)\end{array}$ & $\begin{array}{c}25.5 \\
(20.6)\end{array}$ & $\begin{array}{c}40.9 \\
(47.7)\end{array}$ & $\begin{array}{c}95.2 \\
(26.5)\end{array}$ & $\begin{array}{l}90.0 \\
(7.6)\end{array}$ & $\begin{array}{c}94.7 \\
(19.9)\end{array}$ & $\begin{array}{c}66.8 \\
(20.9)\end{array}$ & $\begin{array}{l}74.5 \\
(5.4)\end{array}$ & $\begin{array}{c}48.7 \\
(22.4)\end{array}$ \\
\hline & Subtotal & 124.2 & 453.1 & 374.2 & 354.9 & 784.1 & $1,027.90$ & $1,153.50$ & 528.8 & 626.2 & 655.4 \\
\hline \multicolumn{12}{|l|}{ Other bacteria } \\
\hline & $15: 0$ & n.m & $\begin{array}{c}19.0 \\
(11.6)\end{array}$ & $\begin{array}{l}10.8 \\
(9.8)\end{array}$ & $\begin{array}{c}9.7 \\
(3.4)\end{array}$ & $\begin{array}{l}11.5 \\
(5.9)\end{array}$ & $\begin{array}{c}6.3 \\
(1.9)\end{array}$ & $\begin{array}{c}6.4 \\
(2.0)\end{array}$ & $\begin{array}{c}29.2 \\
(10.6)\end{array}$ & $\begin{array}{c}33.7 \\
(14.5)\end{array}$ & $\begin{array}{c}46.9 \\
(10.5)\end{array}$ \\
\hline & $16: 1 \omega 7 \mathrm{t}$ & n.m & n.m & n.m & n.m & $\begin{array}{c}42.0 \\
(11.4)\end{array}$ & $\begin{array}{c}54.8 \\
(17.7)\end{array}$ & $\begin{array}{c}55.0 \\
(10.2)\end{array}$ & $\begin{array}{c}85.2 \\
(29.4)\end{array}$ & $\begin{array}{l}106.0 \\
(38.1)\end{array}$ & $\begin{array}{c}74.8 \\
(53.1)\end{array}$ \\
\hline & $17: 0$ & $\begin{array}{l}25.8 \\
(20)\end{array}$ & $\begin{array}{c}30.7 \\
(17.8)\end{array}$ & $\begin{array}{c}26.2 \\
(11.8)\end{array}$ & $\begin{array}{c}25.2 \\
(19.7)\end{array}$ & $\begin{array}{l}46.8 \\
(8.4)\end{array}$ & $\begin{array}{l}45.0 \\
(7.6)\end{array}$ & $\begin{array}{l}59.9 \\
(5.7)\end{array}$ & $\begin{array}{l}34.0 \\
(7.8)\end{array}$ & $\begin{array}{l}31.2 \\
(5.7)\end{array}$ & $\begin{array}{c}56.6 \\
(12.6)\end{array}$ \\
\hline & $18: 1 \omega 9$ & $\begin{array}{c}165.1 \\
(229.2)\end{array}$ & $\begin{array}{c}239.7 \\
(134.8)\end{array}$ & $\begin{array}{l}207.8 \\
(248.2)\end{array}$ & $\begin{array}{c}224.5 \\
(171.8)\end{array}$ & $\begin{array}{l}380.1 \\
(87.4)\end{array}$ & $\begin{array}{l}390.4 \\
(67.0)\end{array}$ & $\begin{array}{l}487.6 \\
(42.0)\end{array}$ & $\begin{array}{l}304.2 \\
(57.7)\end{array}$ & $\begin{array}{l}200.9 \\
(37.8)\end{array}$ & $\begin{array}{c}240.7 \\
(162.0)\end{array}$ \\
\hline & Subtotal & 190.9 & 289.4 & 244.8 & 259.4 & 480.4 & 496.5 & 608.9 & 452.6 & 371.8 & 419.0 \\
\hline & PLFA $^{\text {bacteria }}$ & 346.2 & 864.3 & 727.3 & 803.6 & $1,485.0$ & $1,760.4$ & $2,029.3$ & 1,329.9 & $1,441.1$ & $1,397.3$ \\
\hline \multicolumn{12}{|l|}{$\begin{array}{l}\text { Arbuscular } \\
\text { mycorrhza }\end{array}$} \\
\hline & $16: 1 \omega 5$ & $\begin{array}{c}37.8 \\
(51.9)\end{array}$ & $\begin{array}{c}148.3 \\
(78)\end{array}$ & $\begin{array}{c}170.8 \\
(147.4)\end{array}$ & $\begin{array}{c}77.6 \\
(73.1)\end{array}$ & $\begin{array}{l}30.2 \\
(4.8)\end{array}$ & $\begin{array}{l}33.7 \\
(9.2)\end{array}$ & $\begin{array}{l}51.4 \\
(7.2)\end{array}$ & $\begin{array}{l}25.1 \\
(8.6)\end{array}$ & $\begin{array}{l}16.3 \\
(3.9)\end{array}$ & $\begin{array}{c}46.2 \\
(10.3)\end{array}$ \\
\hline \multicolumn{12}{|l|}{$\begin{array}{l}\text { Actinomycete } \\
\text { genera }\end{array}$} \\
\hline & 10Me18:0 & n.m & n.m & n.m & n.m & $\begin{array}{c}8.7 \\
(1.9)\end{array}$ & $\begin{array}{c}6.2 \\
(2.1)\end{array}$ & $\begin{array}{l}11.3 \\
(1.7)\end{array}$ & $\begin{array}{l}11.3 \\
(2.9)\end{array}$ & $\begin{array}{l}10.1 \\
(4.6)\end{array}$ & $\begin{array}{l}26.0 \\
(5.8)\end{array}$ \\
\hline \multicolumn{12}{|l|}{ Fungal PLFA } \\
\hline & $18: 2 \omega 6,9$ & $\begin{array}{c}242.1 \\
(316.6)\end{array}$ & $\begin{array}{c}564.1 \\
(264.4)\end{array}$ & $\begin{array}{c}425.3 \\
(498.3)\end{array}$ & $\begin{array}{c}568.9 \\
(326.4)\end{array}$ & $\begin{array}{c}852.1 \\
(161.7)\end{array}$ & $\begin{array}{c}1,014.6 \\
(69.3)\end{array}$ & $\begin{array}{l}1,373.4 \\
(118.9)\end{array}$ & $\begin{array}{c}659.7 \\
(204.8)\end{array}$ & $\begin{array}{l}490.8 \\
(91.6)\end{array}$ & $\begin{array}{c}566.1 \\
(396.3)\end{array}$ \\
\hline & PLFA $^{\text {fungal }}$ & 242.1 & 564.1 & 425.3 & 568.9 & 852.1 & $1,014.6$ & $1,373.4$ & 659.7 & 490.8 & 566.1 \\
\hline
\end{tabular}


Table 1. Continued

\begin{tabular}{|c|c|c|c|c|c|c|c|c|c|c|c|}
\hline & & \multirow{3}{*}{ Initial } & \multicolumn{9}{|c|}{ PLFA concentration (nmol/g) } \\
\hline & & & \multicolumn{3}{|c|}{ After 80 days } & \multicolumn{3}{|c|}{ After 160 days } & \multicolumn{3}{|c|}{ After 290 days } \\
\hline & & & pH3 & pH4 & pH5 & pH3 & pH4 & pH5 & pH3 & pH4 & pH5 \\
\hline \multicolumn{12}{|l|}{ Other PLFA } \\
\hline & i14:0 & n.m & $\begin{array}{l}11.5 \\
(7.7)\end{array}$ & $\begin{array}{l}12.3 \\
(9.9)\end{array}$ & $\begin{array}{c}9.5 \\
(4.5)\end{array}$ & $\begin{array}{l}10.1 \\
(6.0)\end{array}$ & $\begin{array}{l}13.0 \\
(3.3)\end{array}$ & $\begin{array}{l}13.1 \\
(4.8)\end{array}$ & $\begin{array}{c}12.0 \\
(17.8)\end{array}$ & $\begin{array}{l}12.9 \\
(5.8)\end{array}$ & $\begin{array}{l}12.4 \\
(2.8)\end{array}$ \\
\hline & $14: 0$ & n.m & $\begin{array}{l}34.6 \\
(23)\end{array}$ & $\begin{array}{c}29.4 \\
(24.6)\end{array}$ & $\begin{array}{c}25.3 \\
(15.2)\end{array}$ & $\begin{array}{c}39.4 \\
(15.2)\end{array}$ & $\begin{array}{l}48.6 \\
(4.2)\end{array}$ & $\begin{array}{c}49.3 \\
(14.1)\end{array}$ & $\begin{array}{l}12.0 \\
(1.8)\end{array}$ & $\begin{array}{l}12.9 \\
(5.8)\end{array}$ & $\begin{array}{l}12.4 \\
(2.8)\end{array}$ \\
\hline & $16: 0$ & $\begin{array}{l}1,126.6 \\
(947.1)\end{array}$ & $\begin{array}{c}975.5 \\
(530.2)\end{array}$ & $\begin{array}{c}726.1 \\
(830.9)\end{array}$ & $\begin{array}{c}792.5 \\
(547.8)\end{array}$ & $\begin{array}{l}1,255.4 \\
(182.4)\end{array}$ & $\begin{array}{l}1,363.6 \\
(185.1)\end{array}$ & $\begin{array}{l}1,660.7 \\
(121.5)\end{array}$ & $\begin{array}{c}950.8 \\
(293.9)\end{array}$ & $\begin{array}{c}928.9 \\
(111.2)\end{array}$ & $\begin{array}{c}983.0 \\
(695.0)\end{array}$ \\
\hline & 18:0 & $\begin{array}{c}715.2 \\
(291.8)\end{array}$ & $\begin{array}{c}201.4 \\
(111.4)\end{array}$ & $\begin{array}{c}130.0 \\
(150.4)\end{array}$ & $\begin{array}{c}200.6 \\
(118.1)\end{array}$ & $\begin{array}{l}312.9 \\
(42.7)\end{array}$ & $\begin{array}{c}314.8 \\
(38)\end{array}$ & $\begin{array}{l}398.5 \\
(30.4)\end{array}$ & $\begin{array}{l}231.6 \\
(46.7)\end{array}$ & $\begin{array}{l}211.4 \\
(15.4)\end{array}$ & $\begin{array}{c}200.0 \\
(126.0)\end{array}$ \\
\hline & $20: 4$ & n.m & n.m & n.m & n.m & $\begin{array}{c}24.2 \\
(21.5)\end{array}$ & $\begin{array}{c}9.5 \\
(3.6)\end{array}$ & $\begin{array}{c}9.3 \\
(1.6)\end{array}$ & $\begin{array}{l}17.9 \\
(2.2)\end{array}$ & $\begin{array}{l}12.2 \\
(2.0)\end{array}$ & $\begin{array}{l}11.0 \\
(2.5)\end{array}$ \\
\hline & $20: 0$ & $\begin{array}{c}23.3 \\
(22.0)\end{array}$ & $\begin{array}{c}58.7 \\
(33.9)\end{array}$ & $\begin{array}{c}22.5 \\
(17.3)\end{array}$ & $\begin{array}{c}20.8 \\
(10.4)\end{array}$ & $\begin{array}{l}30.8 \\
(4.4)\end{array}$ & $\begin{array}{l}30.2 \\
(1.5)\end{array}$ & $\begin{array}{l}48.3 \\
(7.7)\end{array}$ & $\begin{array}{l}21.8 \\
(4.6)\end{array}$ & $\begin{array}{l}21.3 \\
(2.2)\end{array}$ & $\begin{array}{l}33.0 \\
(7.4)\end{array}$ \\
\hline & $21: 0$ & $\begin{array}{c}11.6 \\
(14.9)\end{array}$ & $\begin{array}{c}22.7 \\
(15.3)\end{array}$ & $\begin{array}{c}27.6 \\
(19.6)\end{array}$ & $\begin{array}{l}24.3 \\
(9.2)\end{array}$ & $\begin{array}{l}31.8 \\
(5.4)\end{array}$ & $\begin{array}{l}30.4 \\
(3.2)\end{array}$ & $\begin{array}{c}50.2 \\
(10.3)\end{array}$ & $\begin{array}{l}27.0 \\
(2.6)\end{array}$ & $\begin{array}{l}24.6 \\
(8.0)\end{array}$ & $\begin{array}{c}32.1 \\
(14.2)\end{array}$ \\
\hline & $23: 0$ & $\begin{array}{c}5.4 \\
(1.3)\end{array}$ & $\begin{array}{c}18.0 \\
(10.0)\end{array}$ & $\begin{array}{c}9.6 \\
(2.3)\end{array}$ & $\begin{array}{c}6.5 \\
(0.7)\end{array}$ & $\begin{array}{c}6.2 \\
(1.4)\end{array}$ & $\begin{array}{c}4.8 \\
(0.8)\end{array}$ & $\begin{array}{c}8.4 \\
(1.3)\end{array}$ & $\begin{array}{c}3.0 \\
(0.2)\end{array}$ & $\begin{array}{c}5.0 \\
(0.4)\end{array}$ & $\begin{array}{c}8.2 \\
(1.8)\end{array}$ \\
\hline & $24: 0$ & $\begin{array}{c}8.8 \\
(2.1)\end{array}$ & $\begin{array}{l}16.6 \\
(7.7)\end{array}$ & $\begin{array}{l}18.0 \\
(9.5)\end{array}$ & $\begin{array}{l}16.1 \\
(3.6)\end{array}$ & $\begin{array}{l}12.4 \\
(3.5)\end{array}$ & $\begin{array}{l}10.5 \\
(1.7)\end{array}$ & $\begin{array}{l}16.3 \\
(3.6)\end{array}$ & $\begin{array}{c}8.5 \\
(0.6)\end{array}$ & n.m & n.m \\
\hline & Subtotal & $1,890.9$ & $1,339.0$ & 975.5 & $1,095.6$ & $1,723.2$ & $1,825.4$ & $2,254.1$ & $1,284.6$ & $1,229.2$ & $1,292.1$ \\
\hline & PLFA $^{\text {total }}$ & $2,464.9$ & $2,732.8$ & $2,100.3$ & $2,453.5$ & $4,041.7$ & $4,585.1$ & $5,632.1$ & $3,285.0$ & $3,188.2$ & $3,289.4$ \\
\hline
\end{tabular}

Other bacteria, bacteria other than the Gram+ and Gram-; Other PLFA, PLFA other than the bacterial and fungal PLFA.

Table 2. Shannon-Wiener index, Simpson's Diversity and Evenness (J) of PLFAs in microbial community on the decomposing S. alnifolia leaf litters. Values are means of four replicates, standard deviation are in parentheses

\begin{tabular}{|c|c|c|c|c|c|c|c|c|c|c|}
\hline & \multirow{2}{*}{ Initial } & \multicolumn{3}{|c|}{ After 80 days } & \multicolumn{3}{|c|}{ After 160 days } & \multicolumn{3}{|c|}{ After 290 days } \\
\hline & & pH3 & pH4 & pH5 & pH3 & pH4 & pH5 & pH3 & pH4 & pH5 \\
\hline No. of PLFAs & 14 & 22 & 22 & 22 & 27 & 27 & 27 & 27 & 26 & 26 \\
\hline Shannon's INDEX & $\begin{array}{c}0.788 \\
(0.018)\end{array}$ & $\begin{array}{l}1.043 \\
(0.04)\end{array}$ & $\begin{array}{c}1.037 \\
(0.079)\end{array}$ & $\begin{array}{l}0.999 \\
(0.08)\end{array}$ & $\begin{array}{c}1.075 \\
(0.023)\end{array}$ & $\begin{array}{l}1.037 \\
(0.013)\end{array}$ & $\begin{array}{c}1.026 \\
(0.014)\end{array}$ & $\begin{array}{l}1.023 \\
(0.16)\end{array}$ & $\begin{array}{c}1.062 \\
(0.131)\end{array}$ & $\begin{array}{l}1.001 \\
(0.25)\end{array}$ \\
\hline Evenness & $\begin{array}{c}0.687 \\
(0.015)\end{array}$ & $\begin{array}{l}0.777 \\
(0.03)\end{array}$ & $\begin{array}{c}0.773 \\
(0.059)\end{array}$ & $\begin{array}{l}0.744 \\
(0.06)\end{array}$ & $\begin{array}{c}0.751 \\
(0.071)\end{array}$ & $\begin{array}{c}0.72 \\
(0.003)\end{array}$ & $\begin{array}{l}0.717 \\
(0.01)\end{array}$ & $\begin{array}{c}0.667 \\
(0.112)\end{array}$ & $\begin{array}{c}0.743 \\
(0.093)\end{array}$ & $\begin{array}{c}0.696 \\
(0.177)\end{array}$ \\
\hline Simpson Diversity & $\begin{array}{c}0.704 \\
(0.006)\end{array}$ & $\begin{array}{c}0.791 \\
(0.003)\end{array}$ & $\begin{array}{c}0.782 \\
(0.023)\end{array}$ & $\begin{array}{c}0.796 \\
(0.011)\end{array}$ & $\begin{array}{c}0.818 \\
(0.011)\end{array}$ & $\begin{array}{c}0.818 \\
(0.006)\end{array}$ & $\begin{array}{c}0.818 \\
(0.003)\end{array}$ & $\begin{array}{c}0.853 \\
(0.007)\end{array}$ & $\begin{array}{c}0.862 \\
(0.011)\end{array}$ & $\begin{array}{c}0.864 \\
(0.046)\end{array}$ \\
\hline
\end{tabular}

firming the results of Rechcigl and Sparks (1985), and Wolters (1991a, 1991b). These decreased litter mass losses suggested that acidification inhibits the growth or activities of micro-decomposers, Berg and Laskowski (2006) reported that acidification may affect the decomposition process directly through the effect of $\mathrm{H}^{+}$ions on some decomposers and deterioration of soil conditions for others.

The microbial community structure on the decomposing litters is very complicated and highly sensitive to physico-chemical environmental changes; in addition, there are many methods for identifying soil microbial taxonomic groups and community structures. SIR and PLFAs were comparatively simple methods of quantifying microbial groups. Bååth and Anderson (2003) found good linear correlation between the total microbial biomass estimated with SIR and total amount of PLFAs, indicating that $1 \mathrm{mg}$ biomass-C was equivalent to $130 \mathrm{nmol}$ of total amount of PLFAs. The present results also showed that the 

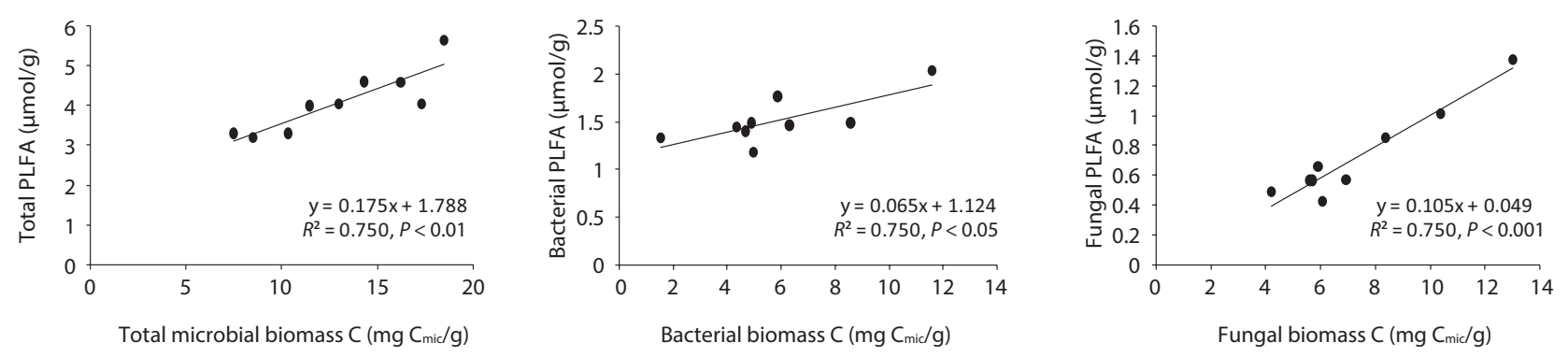

Fig. 5. The relationship between microbial biomass $-\mathrm{C}\left(\mathrm{mg} \mathrm{C}_{\text {mic }} / \mathrm{g}\right)$ by SIR and PLFAs $(\mu \mathrm{mol} / \mathrm{g})$ during litter decomposition in the microcosm.
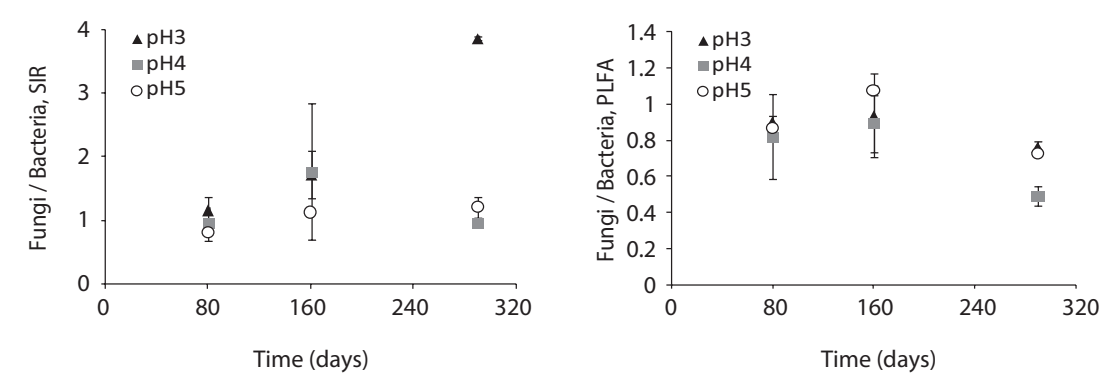

Fig. 6. Changes in fungal-to-bacterial biomass ratio measured by SIR(left) and PLFA(right) during S. alnifolia leaf litter decomposition at different pH treatments in the microcosm environment. The error bars indicate standard deviation.

concentration of PLFAs had positive relationships with microbial biomass of SIR (Fig. 5). Total microbial biomass of SIR and total amount of PLFAs has $R^{2}=0.7509$, and $P<$ 0.001 significant positive relationship, and the $1 \mathrm{mg}$ total microbial biomass estimated with SIR was equivalent to $175 \mathrm{nmol}$ of total PLFAs.

The fungal and bacterial composition determined by SIR and PLFA at the decomposing litter changed by acid treatment and litter decomposition stage (Fig. 6). The fungi/bacteria ratio by SIR and PLFA showed different pattern. These results might be due to the microbial state, for example sporulation, another nonliving deliberation such as humic acid (Nielsen and Petersen 2000), and the ability of response to the nutrient such as glucose (Bailey et al. 2002).

The SIR fungi/bacteria ratio increased throughout the decomposition process; however, at $\mathrm{pH} 4$ the ratio decreased at the late decomposition process. On the other hand, the fungi/bacteria ratio by PLFA was below 1 at low $\mathrm{pH}$ treatments and decomposition stages, though the ratio dramatically decreased at the late stage of decomposition at $\mathrm{pH} 4$ and 5 treatment.

The fungi/bacteria ratio by SIR was higher than that by PLFA which was always below 1 . Especially in low $\mathrm{pH}$ treatment, the fungi/bacteria ratio increased dramatical- ly. Bååth and Anderson (2003) argued that the fungi/bacteria ratio measured using the selective inhibition technique decreased significantly with increasing $\mathrm{pH}$ from about 9 at $\mathrm{pH} 3$ to approximately 2 at $\mathrm{pH}$ 7. These results indicate that low $\mathrm{pH}$ facilitates respiration or growth of microbial groups that are not inhibited by fungal inhibition agent. On the other hand, the fungi/bacteria ratio by PLFA showed smaller differences between $\mathrm{pH}$ treatments as decomposition proceeded. This result demonstrates the fungal predomination at early stage of litter decomposition and bacterial predomination at later decomposition stages.

Bååth et al. (1995), Bååth and Arnebrant (1994), Pennanen et al. (1999), and Arao (1999) also reported that

Table 3. The relationships between litter decomposition and three microbial biomass parameters present in the decaying leaf litter from the different simulated acid rain $\mathrm{pH}$ treatments, taken at $23^{\circ} \mathrm{C}$ and at constant humidity

\begin{tabular}{lccc}
\hline $\begin{array}{c}\text { Microbial biomass by } \\
\text { PLFA (x) }\end{array}$ & $\begin{array}{c}\text { Litter } \\
\text { decomposition }\end{array}$ & $\boldsymbol{R}^{2}$ & $\boldsymbol{P}$-value \\
\hline Total microbial biomass & $2.040 \mathrm{x}+21.53$ & 0.739 & 0.003 \\
Bacterial biomass & $6.498 \mathrm{x}+20.70$ & 0.783 & 0.002 \\
Fungal biomass & $9.580 \mathrm{x}+23.92$ & 0.737 & 0.003 \\
\hline
\end{tabular}

Note: PLFA, phospholipid fatty acid. 
when they increased the $\mathrm{pH}$ of soil by treatment with lime and ash, bacterial biomass and fungal biomass were decreased at higher $\mathrm{pH}$ levels while the fungal biomass decreased by a comparatively larger amount than bacteria at higher $\mathrm{pH}$ levels.

In addition, the PLFAs of each microbial group in the study differed in response to varied $\mathrm{pH}$, similarly to in previous studies (Bååth et al. 1979, Mancinelli 1986, Bewley and Parkinson 1985, Esher et al. 1992, Fritze et al. 1992). Frostegård et al. (1993) found a three-fold increase in the fatty acid 16: 105 in the plots with the highest $\mathrm{pH}$. An increase was also found for the fatty acids i14:0, 16:1 $\omega 9$,

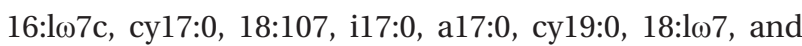
10Mel8:0 and no effect on 18:2 $\omega 6$. In the present study, the

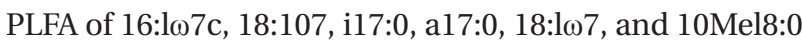
were also increased as $\mathrm{pH}$ increased.

The mass losses of S. anifolia leaf litter by decomposition in a microcosm treated with simulated different $\mathrm{pH}$ acid rain corresponded with decreased of amounts of total microbial biomass, bacterial biomass, and fungal biomass measured by SIR (Lim et al. 2011) and PLFA. The decreased litter decomposition at low $\mathrm{pH}$ was due to the decreased microbial biomass by acidification (Table 3). However, because the decomposer community changes during decomposition stages (i.e., microbial succession), and some microbial groups are supplanted by other groups according to the environmental changes, the decomposition processes are more complicated (Abrahamsen et al. 1980).

There were complementary relationships among microbial groups in the changing environment. Even though the microbial biomasses in the decomposing litter were the same among the different $\mathrm{pH}$ treatment, the microbial species composition (e.g., bacteria to fungi) could be different. Moreover, there is functional redundancy of microbial groups with the changes in environment (Rousk et al. 2009). Changes in species composition within community occurred by environmental changes because each species has its own tolerance range, the acid rain did not significantly change soil $\mathrm{pH}$, and little impact on soil microorganisms and mineralization rate was found (Stemmer et al. 2007). However, most studies reported that there are complementary microbial shifts between bacterial and fungal groups (Rousk and Bååth 2007).

Consequently, the active microbial population dominating in a given soil system is adapted to the conditions of that system, including its $\mathrm{pH}$ (Berg and Laskowski 2006). The conditions of that decomposition subsystem are easily changeable by anthropogenic activities. Acid rain effects soil biotic processes such as $\mathrm{CO}_{2}$ production, microbial biomass in the organic layer, and leaching of $\mathrm{NO}_{3}{ }^{-}$(Wolter 1991a, 1991b, Lim et al. 2011); consequently, the soil acidification due to anthropogenic activities may be too fast for microbial communities to adapt to new, changed conditions (Berg and Laskowski 2006).

\section{LITERATURE CITED}

Abrahamsen G, Hovland J, Hagvar S. 1980. Effects of artificial acid rain and liming on soil organisms and the decomposition of organic matters. In: Effects of Acid Precipitation on Terrestrial Ecosystems (Hutchinson TC, Havas M, eds). Plenum Press, New York, pp 341-362.

Anderson JPE, Domsch KH. 1973. Quantification of bacterial and fungal contributions to soil respiration. Arch Microbiol 93: 113-127.

Anderson JPE, Domsch KH. 1978. A physiological method for the quantitative measurement of microbial biomass in soil. Soil Biol Biochem 10: 215-221.

Arao T. 1999. In situ detection of changes in soil bacterial and fungal activities by measuring ${ }^{13} \mathrm{C}$ incorporation into soil phospholipid fatty acids from ${ }^{13} \mathrm{C}$ acetate. Soil Biol Biochem 31: 1015-1020.

Bååth E, Anderson TH. 2003. Comparison of soil fungal/ bacterial ratios in a $\mathrm{pH}$ gradient using physiological and PLFA-based techniques. Soil Biol Biochem 35: 995-963.

Bååth E, Arnebrant K. 1994. Growth rate and response of bacterial communities to $\mathrm{pH}$ in limed and ash-treated forest soils. Soil Biol Biochem 26: 995-1001.

Bååth E, Frostegård Å, Fritze H. 1992. Soil bacterial biomass, activity, phospholipid fatty acid pattern, and $\mathrm{pH}$ tolerance in an area polluted with alkaline dust deposition. Appl Envir Microb 58: 4026-4031.

Bååth E, Frostegård Å, Pennanen T, Fritze H. 1995. Microbial community structure and $\mathrm{pH}$ response in relation to soil organic matter quality in wood ash fertilized, clear-cut or burned forest soils. Soil Biol Biochem 27: 229-240.

Bååth E, Lundgren B, Söderström B. 1979. Effects of artificial acid rain on microbial activity and biomass. Bull. Environ. Contam Toxicol 23: 737-740.

Bailey VL, Peacock AD, Smith JL, Bolton H Jr. 2002. Relationship between soil microbial biomass determined by chloroform fumigation extraction, substrate-induced respiration, and phospholipid fatty acid analysis. Soil Biol Biochem 34: 1385-1389.

Batty LC, Younger PL. 2007. The effect of $\mathrm{pH}$ on plant litter decomposition and metal cycling in wetland mesocosms supplied with mine drainage. Chemosphere 66: 158-164. 
Beare MH, Neely CL, Coleman DC, Hargrove WL. 1990. A substrate induced respiration (SIR) method for measurement of fungal and bacterial biomass on plant residues. Soil Biol Biochem 22: 585-594.

Berg B, Laskowski R. 2006. Litter decomposition: a guide to carbon and nutrient turnover, Advances in ecological research 38. Elsevier Academic Press, San Diego.

Bewley RJF, Parkinson D. 1985. Bacterial and fungal activity in sulphur dioxide polluted soils. Can J Microbiol 31: 1315.

Esher RJ, Marx DH, Ursic SJ, Baker RL, Brown LR, Coleman DC. 1992. Simulated acid rain effects in fine roots, ectomycorrhizae, microorganisms, and invertebrates in pine forests of the southern United States. Water Air Soil Pollut 61: 269-278.

Evans LS, Gmur NF, Mancini D. 1982. Effects of simulated acidic rain on yields of Raphanus sativus, Lactuca sativa, Triticum aestivum and Medicago sativa. Environ Exp Bot 22: 445-453.

Federle TW. 1986. Microbial distribution in soil: new techniques. In: Perspectives in Microbial Ecology (Megusar F, Gantar M, eds). Slovene Society for Microbiology, Ljublana, pp 493-498.

Fritze H. 1992. Effects of environmental pollution on forest soil microflora. Silva Fennica 26: 37-48.

Fritze H, Kiikkilä O, Pasanen J, Pietikäinen J. 1992. Reaction of forest soil microflora to environmental stress along a moderate pollution gradient next to an oil refinery. Plant Soil 140: 175-182.

Frostegård Å, Bååth E. 1996. The use of phospholipids fatty analysis to estimate bacterial and fungal biomass in soil. Biol Fertil Soils 22: 59-65.

Frostegård A, Bååth E, Tunlio A. 1993. Shifts in the structure of soil microbial communities in limed forest as revealed by phospholipid fatty acid analysis. Soil Biol Biochem 25: 723-730.

Gross S, Robbins EI. 2000. Acidophilic and acid-tolerant fungi and yeasts. Hydrobiologia 433: 91-109.

Heal OW, Anderson JM, Swift MJ. 1997. Plant litter quality and decomposition: an historical overview. In: Driven by Nature: Plant litter Quality and decomposition(Cadisch $\mathrm{G}$, Giller KE, eds). CAB International, Wallingford, pp 3-30.

Hermle S, Vollenweider P, Günthardt-Goerg MS, McQuattie CJ, Matyssek R. 2007. Leaf responsiveness of Populus tremula and Salix viminalis to soil contaminated with heavy metals and acidic rain water. Tree Physiol 27: 1517-1531.

Hovland J, Abrahanmsen G, Ogner G. 1980. Effects of artificial acid rain on decomposition of spruce needles and on mobilisation and leaching of elements. Plant Soil 56: 365-378.

Högberg MN, Högberg P, Myrold DD. 2007, Is microbial community composition in boreal forest soils determined by $\mathrm{pH}, \mathrm{C}$-toN ratio, the trees, or all three? Oecologia 150: 590-601.

Lee JJ, Weber DE. 1979. The effects of simulated acid rain on seedling emergence and growth of eleven woody species. Forest Sci 25: 393-398.

Lim SM, Cha SS, Shim JK. 2011. Effects of simulated acid rain on microbial activities and litter decomposition. J Ecol Field Biol 34: 401-410.

Liu J, Zhou G, Zhang D. 2007. Simulated effects of acidic solutions on element dynamics in monsoon evergreen broad-leaved forest at Dinghushan, China. Part 1: dynamics of K, Na, Ca, Mg and P. Environ Sci Pollut Res Int 14: 123-129.

Mancinelli RL. 1986. Alpine tundra soil bacterial responses to increased soil loading rates of acid precipitation, nitrate, and sulfate Front Range, Colorado, USA. Arctic Alpine Res 18: 269-275.

Matzner E, Murach D, Fortann H. 1986. Soil acidity and its relationship to root growth in declining forest stands in Germany. Water Air Soil Pollut 31: 273-282.

Myrold DD, Nalson GE. 1992. Effect of acid rain on soil microbial processes. In: Environmental Microbiology (Mitchell R, ed). Wiley-Liss Inc., New York, pp 59-81.

Neufold HS, Jernstedt JA, Haines BL. 1985. Direct foliar effects of simulated acid rain I. Damage, growth, and gas exchange. New Phytol 99: 389-405.

Nielsen P, Petersen SO. 2000. Ester-linked polar lipid fatty acid profiles of soil microbial communities: a comparison of extraction methods and evaluation of interference from humic acids. Soil Biol Biochem 32: 1241-1249.

Osonbui O, Oren R, Werk K, Schulze ED, Heilmeier H. 1988. Performance of two Picea abies (L.) Karst stands at different stages of decline IV. Xylem sap concentration of magnesium, calcium, potassium, and nitrogen. Oecologia $77: 1-6$.

Ouyang XJ, Zhao GY, Huang ZL, Liu JX, Zhang DQ, Li J. 2008. Effect of Simulated acid rain on potential carbon and nitrogen mineralization in forest soils. Pedosphere 18: 503-514.

Pennanen T, Fritze H, Vanhala P, Kiikkila O, Neuvonen S, Bååth E. 1998. Structure of a microbial community in soil after prolonged addition of low levels of simulated acid rain. Appl Environ Microbiol 64: 2173-2180.

Pennanen T, Liski J, Bååth E, Kitunen V, Uotila J, Westman CJ, Fritze H. 1999. Structure of the microbial communities in coniferous forest soils in relation to site fertility 
and stand development stage. Microbiol Ecol 38: 168179.

Proctor JTA. 1983. Effect of simulated acid sulfuric rain on apple tree foliage, nutrient content, yield, and fruit quality. Environ Exp Bot 23: 167-174.

Raynal DJ, Roman JR, Eichelaub WM. 1982. Response of tree seedlings to acid precipitation II. Effect of simulated canopy throughfall on sugar maple seedling growth. Environ Exp Bot 22: 385-392.

Rechcigl JE, Sparks DL. 1985. Effect or acid rain on the soil environment: a review. Commun Soil Sci Plant Anal 16: 653-680.

Reich PB, Scholettle AW, Stroo HF, Troiano J, Amundson RG. 1987. Effects of ozone and acid rain on white pine (Pinus strobes) seedlings grown in five soils. I. Net photosynthesis and growth. Can J Bot 65: 977-987.

Reith F, Drake HL, Kusel K. 2002. Anaerobic activities of bacteria and fungi moderately acidic conifer and deciduous leaf litter. FEMS Microbiol Ecol 41: 27-35.

Rousk J, Bååth E. 2007. Fungal biomass production and turnover in soil estimated using the acetate-in-ergosterol technique. Soil Biol Biochem. 39: 2173-2177.

Rousk J, Brookkes P, Bååth E. 2009 Contrasting soil pH effects on fungal and bacterial growth suggest functional redundancy in carbon mineralization. Appl Environ Microbiol 75: 1589-1596.

Sariyildiz T, Anderson JM. 2003. Interactions between litter quality, decomposition and soil fertility: a laboratory study. Soil Biol Biochem 35: 391-399.

Shannon CE. 1948. A mathematical theory of communication. Bell Syst Tech J 27: 379-423, 623-656.

Simpson EH. 1949. Measurement of diversity. Nature 163:
688.

Stemmer M, Watzinger A, Blochberger K, Haberhauer G., Gerzabek MH. 2007, Linking dynamics of soil microbial phospholipid fatty acids to carbon mineralization in a ${ }^{13} \mathrm{C}$ natural abundance experiment: Impact of heavy metals and acid rain. Soil Biol Biochem 39: 3177-3186.

Wilkinson SC, Anderson JM, Scardelis SP, Tisiafouli M, Taylor A, Wolters V. 2002. PLFA profiles of microbial communities in decomposing conifer litters subject to moisture stress. Soil Biol Biochem 34: 189-200.

Wolters V. 1991a. Effects of acid rain on leaf-litter decomposition in a beech forest on calcareous soil. Biol Fertil Soils 11: 151-156.

Wolters V. 1991b. Biological processes in two beech forest soils treated with simulated acid rain - a laboratory experiment with Isotoma tigrina (Insecta, Collembola). Soil Biol Biochem 23: 381-390.

Wood M, Cooper JE, Holding AJ. 1984 Aluminum toxicity and nodulation of Trifolium repens. Plant Soil 78: 381-391.

Wood T, Bormann FH. 1974. Effects of an artificial acid mist upon the growth of Bestula alleghaniensis. britt. Environ Pollut 7: 256-298.

You YH, Lee CS, Kim JH. 1998. Selection of Tolerant Species among Korean Major Woody Plants to Restore Yeocheon Industrial Complex Area. Korean J Ecol 21: 337-344.

Zhang JE, Ouyang Y, Ling DJ. 2007. Impacts of simulated rain on cation leaching from the Latosol in South China. Chemosphere 67: 2131-2137.

Zimmer M. 2002. Is decomposition of woodland leaf litter influenced by its species richness?. Soil Biol Biochem 34: 277-284. 